\title{
Valores críticos de densidade do solo avaliados por condições de contorno
}

\author{
Critical values of soil bulk density evaluated by boundary conditions
}

Paulo Ivonir Gubiani ${ }^{{ }^{*}}$ Dalvan José Reinert ${ }^{\mathrm{I}}$ José Miguel Reichert ${ }^{\mathrm{I}}$

RESUMO

A associação irregular entre produção das culturas e densidade do solo ( $\rho$ ) dificulta a aplicação da $\rho$ para diagnóstico e manejo da compactação do solo. Com o objetivo de analisar esta problemática, este trabalho apresenta um procedimento matemático de simulação de limites de $\rho$ por demanda hídrica e a dinâmica da resistência do solo à penetração $(R)$ em função do tempo ( $t$ ) de extração de água por evapotranspiração (E) da camada de solo ocupada pelo sistema radicular $(L)$. Parâmetros de curvas de $R$ e de retenção de água de um Latossolo foram utilizados nas simulações de $\rho$ e $R$ para diferentes condições de $E$, t e L. A $\rho$ variou inversamente proporcional à $E$ e t e diretamente proporcional à $L$. A equação para cálculo da $\rho$ possibilitou, também, calcular a densidade crítica $\left(\rho_{c}\right)$ obtida com o intervalo hídrico ótimo. Simulações para até seis dias de extração de água $(t=6)$ de camadas de solo L com $\rho$ igual à $\rho$ para o solo deste estudo $\left(1340 \mathrm{~kg} \mathrm{~m}^{-3}\right)$ indicam que a $R$ máxima pode aumentar de 2 para $8 \mathrm{MPa}$, dependendo das condições de $E$ (de 0,002 a 0,006m $d^{-1}$ ) e $L$ (de 0,2 a 0,6m). Baixos valores de $R$ obtidos quando a $E$ é pequena e L é grande caracterizam condições em que plantas podem produzir normalmente, mesmo que a $\rho$ seja igual a $\rho_{c}$.

Palavras-chave: compactação, evapotranspiração, modelagem, manejo do solo.

\section{ABSTRACT}

The irregular association between crop production and soil bulk density $(\rho)$ weakens the application of $\rho$ for diagnosis and management of soil compaction. With the aim of evaluating this problem, this study introduce a mathematical procedure to simulate limits of $\rho$ for water demand and soil penetration resistance $(R)$ dynamic as a function of the time $(t)$ of water extraction by crop evapotranspiration $(E)$ of the soil layer explored by plant roots $(L)$. Parameters of both penetration resistance and water retention curves of an Oxisol were used in the simulations of $\rho$ and $R$ for different conditions of $E$, $t$ and $L$. The $\rho$ varied inversely proportional to $E$ and $t$ and directly proportional to $L$. The equation of $\rho$ also allows calculating the critical bulk density $\left(\rho_{e}\right)$ calculated with the least limit water range (LLWR). Simulations done for up to six days of water extraction $(t=6)$ in soil layers $L$ with $\rho_{c}$ from the LLWR for the Oxisol $\left(1340 \mathrm{~kg} \mathrm{~m}^{-3}\right)$ indicate that the maximum $R$ may increase from 2 to $8 M P a$, depending upon the $E(0.002$ to $\left.0.006 \mathrm{~m} \mathrm{~d}^{-1}\right)$ and $L(0.2$ to $0.6 \mathrm{~m})$ conditions. Low values of $R$ obtained when the $E$ is small and $L$ is large characterize conditions for normal crops production even the $\rho$ is equal to the $\rho_{c}$.

Key words: soil compaction, evapotranspiration, modeling, soil management.

\section{INTRODUÇÃo}

As alterações provocadas pelo uso agrícola na distribuição de poros, nas propriedades mecânicas e em processos de transporte de água e gases no solo estão direta ou indiretamente relacionadas com a densidade do solo $(\rho)$, motivo pelo qual ela é usada como termo independente no intervalo hídrico ótimo (IHO) (SILVA et al., 1994) e como indicadora indireta de restrições físicas ao crescimento de plantas (LETEY, 1985; REICHERT et al., 2003). A densidade do solo crítica para o crescimento de plantas $\left(\rho_{\mathrm{c}}\right)$ tem sido definida como a densidade que corresponde ao valor zero do IHO. Para um solo, há apenas um valor de $\rho_{c}$, a não ser que sejam modificados os limites do IHO deste solo (KAISER et al., 2009).

Compilando resultados de pesquisas em que o IHO foi definido por limites de resistência à penetração de $2 \mathrm{MPa}$, porosidade de aeração de $10 \%$, capacidade de campo a $10 \mathrm{kPa}$ e ponto de murcha

IDepartamento de Solos, Centro de Ciências Rurais (CCR), Universidade Federal de Santa Maria (UFSM), 97105-900, Santa Maria, RS, Brasil. E-mail: paulogubiani@gmail.com.*Autor para correspondência. 
permanente a $1500 \mathrm{kPa}$, REICHERT et al. (2009) demonstraram que a densidade crítica $\left(\rho, \mathrm{kg} \mathrm{m}^{-3}\right)$ pode ser estimada em função da fração argila do solo $\left(\mathrm{A}, \mathrm{kg} \mathrm{kg}^{-1}\right)$ por uma equação linear $\rho_{\mathrm{c}}=\mathrm{aA}+\mathrm{b}$, com $\mathrm{a}=-0,78 \mathrm{~kg} \mathrm{~m}^{-3}$ e $\mathrm{b}=1838 \mathrm{~kg} \mathrm{~m}^{-3}\left(\mathrm{r}^{2}=0,92\right)$. Contudo, discordâncias entre resposta de plantas verificada e esperada para solos com densidades maiores que a $\rho_{c}$ motivaram a suposição que os limites do intervalo hídrico ótimo precisam ser ajustados e a conclusão de que a $\rho_{c}$ não é restritiva, mas representa densidade próxima da restritiva ao crescimento e produção das culturas (REICHERT et al., 2009).

Além das inúmeras pesquisas sumarizadas no trabalho de REICHERT et al. (2009), resultados de oito cultivos de milho em solos com diferentes níveis de compactação mostraram não haver relação entre produção de grãos e densidade do solo, embora as densidades cobrissem uma ampla faixa em torno da $\rho_{c}$ (GUBIANI, 2012). Em havendo tais evidências factuais, explicita-se o problema de irregularidade da associação entre resposta de plantas e densidade do solo, algo implicitamente presente na definição de fator indireto de restrições físicas ao crescimento de plantas (LETEY, 1985; REICHERT et al., 2003). Essas evidências sugerem que a associação entre resposta biológica e densidade do solo é circunstanciada por fatores internos e externos à estrutura do solo, os quais controlam fatores diretos como resistência mecânica, água e oxigênio.

Como a evapotranspiração determina a extração de água do solo e a profundidade do sistema radicular determina a camada que perde água, então a taxa de aumento da resistência mecânica depende desses fatores, visto que a resistência mecânica é função direta do conteúdo de água (LETEY, 1985). Além disso, evapotranspiração, profundidade do sistema radicular e conteúdo de água do solo não são determinados diretamente pela densidade do solo. Por conseguinte, há grande variabilidade da resistência mecânica para um dado grau de compactação do solo ou coincidência de valores para graus de compactação diferentes (SILVA et al., 2008).

Decorre disso que o crescimento radicular pode ser ou não afetado em densidades menores, iguais ou maiores que a $\rho_{c}$, dependendo das circunstâncias de demanda e oferta hídrica. Assim, a densidade que uma camada de solo deve ter para garantir que a extração de água acumulada num período de tempo definido mantenha a resistência mecânica do solo menor que um valor máximo estabelecido pode ser diferente em cada condição particular de cultivo.
O conhecimento da demanda hídrica é complexo e requer a descrição de processos de fluxo de água no sistema solo-planta-atmosfera (JONG VAN LIER et al., 2008). Entretanto, o uso de estados desses processos dentro dos seus limites físicos é uma estratégia útil para investigar como eles determinam os limites de densidade e a dinâmica da resistência mecânica. Nesse contexto, o objetivo deste trabalho foi simular limites de densidade em função da taxa e do tempo de extração de água, e da espessura da camada ocupada pelo sistema radicular, bem como avaliar o efeito desses fatores na dinâmica da resistência mecânica em função da demanda hídrica.

\section{MATERIAL E MÉTODOS}

Amostras de solo com estrutura preservada, num total de 515, foram coletadas em cilindros metálicos de $0,05 \mathrm{~m}$ de altura e $0,06 \mathrm{~m}$ de diâmetro, nas profundidades de $0,07 \mathrm{~m}$ e $0,25 \mathrm{~m}$, em um Latossolo Vermelho Distroférrico típico $\left(0,12 \mathrm{~kg} \mathrm{~kg}^{-1}\right.$ de areia, $0,24 \mathrm{~kg} \mathrm{~kg}^{-1}$ de silte e $0,64 \mathrm{~kg} \mathrm{~kg}^{-1}$ de argila), situado em Ijuí-RS (28 26’30,26”S, 5400’58,31W, altitude $300 \mathrm{~m})$. Ampla variação de densidade do solo $(1020 \mathrm{~kg}$ $\mathrm{m}^{-3}$ a $1470 \mathrm{~kg} \mathrm{~m}^{-3}$ ) foi obtida com a coleta de amostras em diferentes níveis de compactação, constituídos pela condição atual da área sob plantio direto, plantio direto seguido de escarificação e plantio direto com aplicação de compactação adicional por tráfego (detalhes em GUBIANI, 2012). As amostras foram saturadas e drenadas sob tensões $(\psi)$ de 1, 6, 10, 33 e $100 \mathrm{kPa}$. Após a drenagem, aproximadamente 85 amostras provenientes de pontos de coleta com diferentes estados de compactação foram retiradas em cada $\psi$, pesadas e submetidas ao teste de resistência à penetração. Outras 94 amostras permaneceram expostas ao ar para diminuição do conteúdo de água por evaporação a fim de se obter outros dois grupos de 47 amostras cada um, com conteúdo de água diferente e menor que o das amostras drenadas até $100 \mathrm{kPa}$. $\mathrm{O}$ conteúdo de água de cada grupo foi monitorado por pesagem para que a $\psi$ ficasse em torno de $500 \mathrm{kPa}$ e $1500 \mathrm{kPa}$. Nesse intervalo de tensões, a relação conteúdo de água versus $\psi$ já era conhecida para este solo, por meio de medições com psicrômetro de ponto de orvalho - WP4 $[\theta=\mathrm{f}(\psi)$, com $\mathrm{R}^{2}=0,989$ e $\left.\left.\mathrm{P}<0,0001\right)\right]$. Como não há necessidade de informar a $\psi$ no modelo de resistência à penetração usado neste trabalho (BUSSCHER, 1990), os valores de $500 \mathrm{kPa}$ e $1500 \mathrm{kPa}$ apenas indicam que a $\mathrm{R}$ foi medida até conteúdos de água próximos aos do ponto de murcha permanente. Cessado o período de evaporação, as amostras foram transferidas para 
dentro de embalagem plástica, onde foram mantidas por $72 \mathrm{~h}$ para redistribuição e homogeneização do conteúdo de água.

A resistência do solo à penetração ( $R$, $\mathrm{MPa}$ ) foi medida com um penetrômetro eletrônico de bancada, com haste metálica, cone de $4 \mathrm{~mm}$ de diâmetro na base e ângulo de $30^{\circ}$ a uma velocidade constante de $10 \mathrm{~mm} \mathrm{~min}^{-1}$. Na sequência, as amostras foram secas a $105^{\circ} \mathrm{C}$ até massa constante para cálculo da densidade do solo $\left(\rho, \mathrm{kg} \mathrm{m}^{-3}\right)$ e do conteúdo volumétrico de água $\left(\theta, \mathrm{m}^{3} \mathrm{~m}^{-3}\right)$. Aos dados de $\mathrm{R}$, $\rho$ e $\theta$ foi ajustado o modelo de BUSSCHER (1990) (Eq. 1). Este modelo foi escolhido porque, das principais variáveis utilizadas para explicar a $\mathrm{R}(\rho, \theta$, granulometria e matéria orgânica do solo), se obteve variação apenas da $\rho$ e do $\theta$ na área experimental. Em solos com ampla variação de carbono, como o estudado por TAVARES FILHO et al. (2012), pode haver efeito da matéria orgânica na $\mathrm{R}$ e o modelo de estimativa da $\mathrm{R}$ pode incluir essa variável preditora.

$$
\mathrm{R}=\mathrm{m} \rho^{\mathrm{n}} \theta^{\mathrm{k}}
$$
adimensionais.

Em que: $m=M P a m^{3 n} k^{-n}$, e $n$ e $k$ são

Para atribuir $\mathrm{o}$ efeito de fatores meteorológicos e de espécies vegetais à variação do $\theta$, o $\theta$ foi substituído por outras variáveis. $O$ conteúdo volumétrico de água $\theta$ num instante t qualquer pode ser representado subtraindo-se, do conteúdo volumétrico de água na saturação $\left(\theta_{\mathrm{s}}\right)$, a parcela volumétrica de água extraída do solo $\left(\theta_{\mathrm{e}}\right)$ até o instante t considerado, isto é:

$$
\theta=\theta_{\mathrm{s}}-\theta_{\mathrm{e}}
$$

Para um período sem precipitação, a mudança de $\theta_{\mathrm{e}}$ que ocorre numa camada de solo L (m) se deve apenas a saídas de água da camada. Assim, a mudança diária de $\theta_{\mathrm{e}}$ até o instante $\mathrm{t}(\mathrm{d})$ pode ser representada pelo somatório das saídas de água por evapotranspiração real $\left(\mathrm{E}, \mathrm{m} \mathrm{d} \mathrm{d}^{-1}\right), \sum_{\mathrm{i}=1}^{\mathrm{n}} \mathrm{E}_{\mathrm{i}} \Delta \mathrm{t}$, e drenagem $\left(\mathrm{D}, \mathrm{m} \mathrm{d}^{-1}\right), \sum_{\mathrm{i}=1}^{\mathrm{n}} \mathrm{D}_{\mathrm{i}} \Delta \mathrm{t}$, sendo $\mathrm{i}=1,2, \ldots$, $\mathrm{n}$ dias e $\Delta \mathrm{t}=1$ dia:

$$
\theta_{\mathrm{e}}=\left(\frac{\sum_{\mathrm{i}=1}^{\mathrm{n}} \mathrm{D}_{\mathrm{i}} \Delta \mathrm{t}+\sum_{\mathrm{i}=1}^{\mathrm{n}} \mathrm{E}_{\mathrm{i}} \Delta \mathrm{t}}{\mathrm{L}}\right)
$$

Simplificando e substituindo a equação 3 na equação 2 ,

$$
\theta_{\mathrm{i}}=\theta_{\mathrm{s}}-\left(\frac{\left(\sum_{\mathrm{i}=1}^{\mathrm{n}} \mathrm{D}_{\mathrm{i}}+\sum_{\mathrm{i}=1}^{\mathrm{n}} \mathrm{E}_{\mathrm{i}}\right) \Delta \mathrm{t}}{\mathrm{L}}\right)
$$

resulta:

e substituindo a equação 4 na equação 1 ,

$$
\mathrm{R}=\mathrm{m} \rho^{\mathrm{n}}\left(\theta_{\mathrm{s}}-\left(\frac{\left(\sum_{\mathrm{i}=1}^{\mathrm{n}} \mathrm{D}_{\mathrm{i}}+\sum_{\mathrm{i}=1}^{\mathrm{n}} \mathrm{E}_{\mathrm{i}}\right) \Delta \mathrm{t}}{\mathrm{L}}\right)\right)^{\mathrm{k}}
$$

Assumindo que $\sum_{\mathrm{i}=1}^{\mathrm{n}} \mathrm{D}_{\mathrm{i}} \Delta \mathrm{t}$ representa a diferença de conteúdo de água entre a saturação $\left(\theta_{\mathrm{s}}\right)$ e a capacidade de campo $\left(\theta_{\text {cc }}\right)$ da camada L, resulta:

$$
\sum_{\mathrm{A} \text { acurácea da equação }}^{\mathrm{n}} \mathrm{D}_{\mathrm{i}} \Delta \mathrm{t}=\left(\theta_{\mathrm{s}}-\theta_{\mathrm{c}}\right) \mathrm{L}
$$

(6) depende do quanto a estimativa do $\theta_{\text {cc }}$ corresponde a um conteúdo de água para o qual o fluxo descendente pode ser considerado desprezível como componente de diminuição do armazenamento. Nesse sentido, TWARAKAVI et al. (2009) desenvolveram uma estratégia de estimativa do $\theta_{\text {cc }}$ com base em limites de fluxo de drenagem, o que melhoraria a estimativa de drenagem feita com a equação (6).

Substituindo a equação 6 na equação 5 e fazendo as simplificações, resulta:

$$
R=m \rho^{n}\left(\theta_{c}-\frac{\sum_{i=1}^{n} E_{i} \Delta t}{L}\right)^{k}
$$

Com o propósito de avaliar a mudança na $R$ provocada pela $E$, e considerando a $E$ média $\left(E_{m}\right)$ para determinado período de tempo t, a equação (7) é reescrita para:

$$
\mathrm{R}=\mathrm{m} \rho^{\mathrm{n}}\left(\theta_{\mathrm{c}}-\frac{\mathrm{E}_{\mathrm{m}} \mathrm{t}}{\mathrm{L}}\right)^{\mathrm{k}}
$$

Resolvendo, para densidade $\rho$, a equação (8) é rearranjada para:

$$
\rho=\left\{\frac{R}{\left(\theta_{c}-\frac{E_{m} t}{L}\right)^{k} m}\right\}^{\frac{1}{n}}
$$

Estabelecendo-se um valor fixo de R, a $\rho$ pode ser calculada em função das demais variáveis. $\mathrm{Na}$ equação (9), R, $\mathrm{E}_{\mathrm{m}}$, t e L podem ser considerados independentes. Entretanto, o $\theta_{c c}$ estimado em laboratório, comumente na tensão $(\psi)$ de $10 \mathrm{kPa}$, é afetado pela $\rho$ (SILVA et al., 1994), o que determina que a equação (9) não tenha solução exata. Assim, o procedimento de cálculo deve ser iterativo, começando-se com um valor inicial arbitrário de $\rho$, calcular $\theta_{c c}=\mathrm{f}(\psi, \rho)$, introduzir $\theta_{c c}$ na equação (9) e obter o novo valor de $\rho$. Esse procedimento deve ser repetido até que as diferenças entre $\rho_{\mathrm{i}}$ e $\rho_{\mathrm{i}+1}$ sejam menores que um valor para o qual as diferenças podem ser consideradas desprezíveis (por exemplo: $\left.\left|\rho_{\mathrm{i}}-\rho_{\mathrm{i}+1}\right|<0,0001\right)$. O $\theta_{\mathrm{cc}}$ em função da densidade foi considerado como o conteúdo volumétrico de água retido na tensão de $10 \mathrm{kPa}$, ajustado em função da $\rho$ com um modelo quadrático: 
$\theta_{\mathrm{cc}}=\mathrm{q} \rho^{2}+\mathrm{r} \rho+\mathrm{s}$

Em que: $\mathrm{q}=\mathrm{m}^{6} \mathrm{~kg}^{-2}, \mathrm{r}=\mathrm{m}^{3} \mathrm{~kg}^{-1}$ e $\mathrm{s}=\mathrm{m}^{3} \mathrm{~m}^{-3}$.

\section{RESULTADOS E DISCUSSÃO}

O ajuste do modelo de BUSSCHER (1990) para a curva de R e do modelo quadrático para o $\theta_{\text {cc }}$ foi significativo $(\mathrm{P}<0,001)$ e, em ambos os ajustes, os coeficientes das equações (Tabela 1) foram significativos pelo teste $\mathrm{t}(\mathrm{P}<0,001)$. O modelo quadrático indicou que o $\theta_{\text {cc }}$ aumentou até a densidade de $1330 \mathrm{~kg} \mathrm{~m}^{-3}$, decrescendo a partir desse valor. Essa distribuição é coerente, visto que um solo com baixa densidade é poroso, e o aumento da densidade converte poros grandes em poros pequenos, aumentando o volume de poros que permanecem preenchidos com água na tensão $10 \mathrm{kPa}$. Esse processo prossegue a taxas decrescentes até o limite a partir do qual o aumento da densidade provoca diminuição também no volume de poros que permanecem preenchidos com água. Essa distribuição é mais representativa das alterações no tamanho de poros e, portanto, mais apropriada que o aumento linear do conteúdo de água na capacidade de campo com o aumento da densidade, usado por diversos autores na confecção do IHO.

Com a inclusão no conjunto de dados medidos do conteúdo de água calculado para porosidade de aeração de $10 \%$ ( $\theta$ pa) em densidades maiores que as medidas $\left(1480 \mathrm{~kg} \mathrm{~m}^{-3}\right)$, uma única equação quadrática descreveu tanto $0 \theta_{c c}$ como o $\theta$ pa (Figura 1a). Por isso, ao invés de usar a equação (10), optou-se por usar a equação (11), cujo ajuste foi significativo $(\mathrm{P}<0,001)$ e os coeficientes significativos pelo teste $\mathrm{t}(\mathrm{P}<0,001)$ (Tabela 1$)$. A equação (11) considera o limite de $\theta$ pa no cálculo da densidade, mesmo que ele não tivesse sido explicitamente incluído na equação (9).

$\theta_{\text {cc:pa }}=\mathrm{u} \rho^{2}+\mathrm{v} \rho+\mathrm{w}$

Em que: $\mathrm{u}=\mathrm{m}^{6} \mathrm{~kg}^{-2}, \mathrm{v}=\mathrm{m}^{3} \mathrm{~kg}^{-1} \mathrm{e} \mathrm{w}=\mathrm{m}^{3} \mathrm{~m}^{-3}$.

A aplicação da equação (9) com o uso das equações (1) e (11) foi exemplificada para
$\mathrm{E}_{\mathrm{m}}$ de $0,003 \mathrm{~m} \mathrm{~d}^{-1}$ (Figura 1b) e $\mathrm{E}_{\mathrm{m}}$ de $0,005 \mathrm{~m} \mathrm{~d}^{-1}$ (Figura 1d), para $\mathrm{R}$ crítica de $2 \mathrm{MPa}$, variando-se a camada de extração de água (L) e o tempo (t) para a $\mathrm{R}$ atingir os valores de $2 \mathrm{MPa}$. Com o algoritmo de cálculo escrito em VBA (disponivel em http:// gubianisolos.blogspot.com.br/), podem ser feitas simulações com outros valores de $E_{m}$ e $R$ e com outros modelos da curva de $\mathrm{R}$ e conteúdo de água. Auxílio sobre o uso e modificação do algoritmo pode ser solicitado aos autores deste trabalho.

A $\rho$ calculada com a equação (9) é inversamente proporcional a $\mathrm{E}_{\mathrm{m}}$ e ao período de extração de água (t) e diretamente proporcional à profundidade do sistema radicular (L) e ao valor máximo estabelecido de $\mathrm{R}$ (Figura 1b,d). Consequentemente, as restrições mecânicas são menos recorrentes quando o curso de extração de água do solo progride a taxas menores. Assim, um mesmo grau de compactação do solo pode ter implicações diferentes em cultivos semeados no início ou no final do calendário de semeadura ou em regiões fisiográficas diferentes, dada as diferenças de precipitação e evapotranspiração entre as épocas e regiões. Ainda, evapotranspirações semelhantes podem ocorrer para espécies vegetais diferentes, cuja implicação na extração de água no solo está associada ao aprofundamento do sistema radicular de cada espécie (CARLESSO, 1995). Nesses casos, a equação (9) indica que a progressão da resistência mecânica para níveis máximos estabelecidos será mais lenta para as espécies com maior aprofundamento de raízes.

A análise da equação (9) fornece duas observações importantes. A primeira é que, para $\mathrm{t}=0$, ela se reduz à equação (1) resolvida para $\rho$ no teor de água na capacidade de campo. Nesse caso, ela fornece a $\rho$ para a qual se interceptam as curvas do conteúdo de água, da resistência à penetração crítica e do conteúdo de água na capacidade de campo num gráfico do IHO (COLLARES et al., 2006; KAISER et al., 2009). Como há evidências regulares das pesquisas de que o ponto de murcha permanente não é um limite do IHO que determina o valor da

Tabela 1 - Valores de ajuste dos coeficientes das equações.

\begin{tabular}{|c|c|c|c|c|c|c|c|c|}
\hline \multirow{2}{*}{\multicolumn{3}{|c|}{$\begin{array}{c}\text { Equação (1) } \\
\mathrm{R}=\mathrm{m} \rho^{\mathrm{n}} \theta^{\mathrm{k}}\end{array}$}} & \multicolumn{3}{|c|}{ Equação (10) } & \multicolumn{3}{|c|}{ Equação (11) } \\
\hline & & & \multicolumn{3}{|c|}{$\theta_{\mathrm{cc}}=\mathrm{q} \rho^{2}+\mathrm{r} \rho+\mathrm{s}$} & \multicolumn{3}{|c|}{$\theta_{\text {cc:pa }}=u \rho^{2}+v \rho+w$} \\
\hline $\mathrm{m}$ & $\mathrm{n}$ & $\mathrm{k}$ & $\mathrm{q}$ & $\mathrm{r}$ & $\mathrm{s}$ & $\mathrm{u}$ & $\mathrm{v}$ & $\mathrm{w}$ \\
\hline $7,0310^{-20}$ & 5,9845 & $-1,9921$ & $-8,8710^{-7}$ & $2,3610^{-3}$ & $-1,11799$ & $-1,010^{-6}$ & $2,6410^{-3}$ & $-1,3506$ \\
\hline \multicolumn{3}{|c|}{$\mathrm{N}=515, \mathrm{R}^{2}=0,67$} & \multicolumn{3}{|c|}{$\mathrm{N}=96, \mathrm{R}^{2}=0,33$} & \multicolumn{3}{|c|}{$\mathrm{N}=109, \mathrm{R}^{2}=0,47$} \\
\hline
\end{tabular}

$\mathrm{N}$ : tamanho da amostra. 


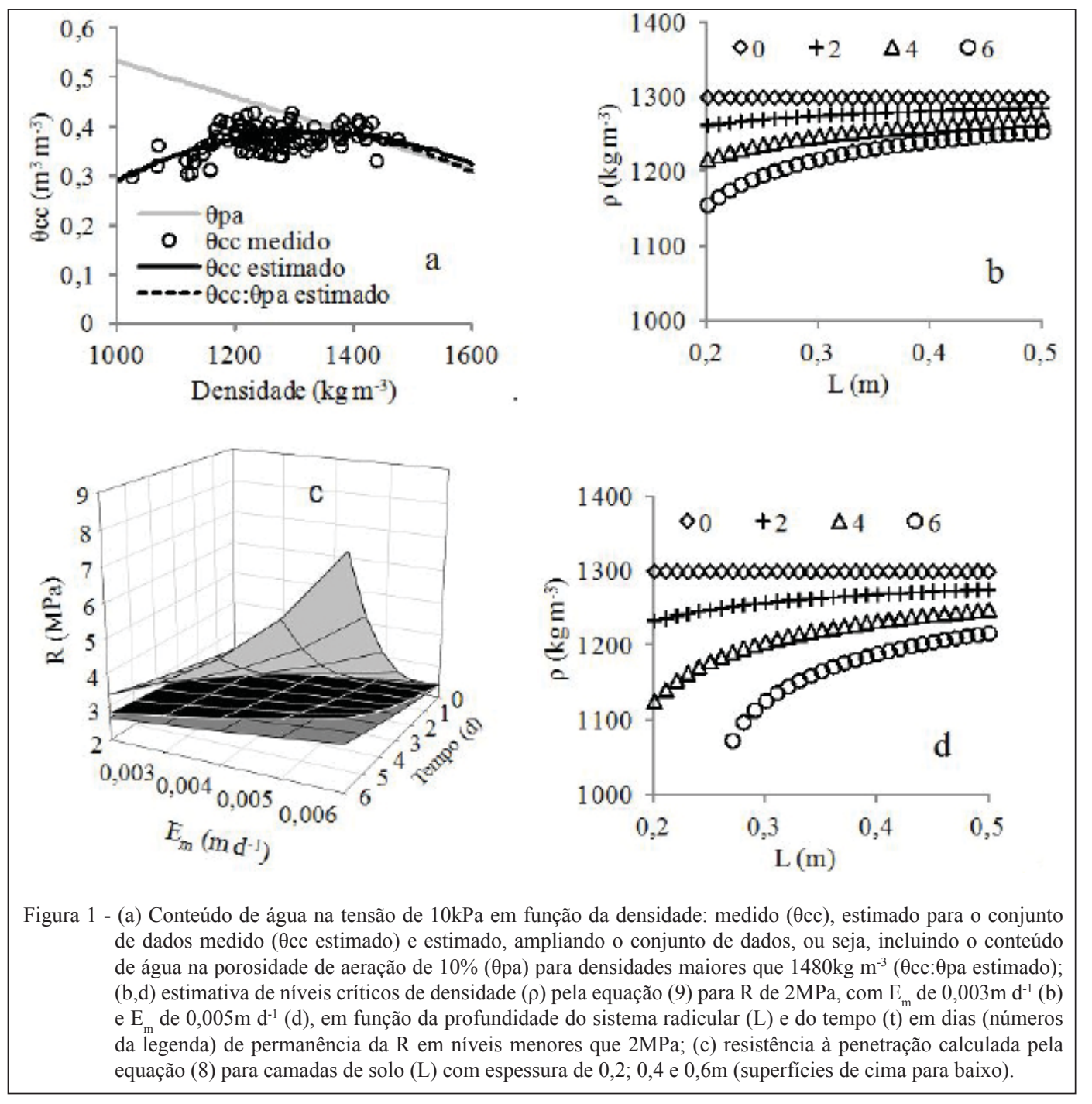

$\rho_{c}$, então a $\rho$ calculada com equação (9) para $t=0$ corresponde à $\rho_{c}$ que seria obtida se fosse gerado o IHO. Neste estudo, a $\rho$ de $1300 \mathrm{~kg} \mathrm{~m}^{-3}$ para $\mathrm{t}=0$ foi semelhante à $\rho_{c}$ de $1340 \mathrm{~kg} \mathrm{~m}^{-3}$, calculada com a equação de REICHERT et al. (2009). A exclusão do efeito da taxa de extração de água e da profundidade do sistema radicular quando $\mathrm{t}=0$ é ilustrado pela linha paralela à abscissa nas figuras $1 \mathrm{~b}$ e $1 \mathrm{~d}$. Portanto, a equação (9) indica que um valor crítico de densidade do solo depende de outros fatores além dos que estão contemplados na atual proposição de IHO.

Em decorrência disso, a segunda observação é que a $\rho$ calculada com a equação (9) é menor ou, no máximo, igual à $\rho_{\mathrm{c}}$, ambas calculadas com base no mesmo valor de R. A $\rho$ só será maior que a $\rho_{c}$ se, no cálculo da $\rho$, a R for maior que no cálculo da $\rho_{c}$. Guardadas essas condições, a $\rho$ será gradativamente afastada para valores menores que a $\rho_{\mathrm{c}}$ quanto maior o valor de $\mathrm{t}$ (Figuras 1b,d) e de $\mathrm{E}_{\mathrm{m}}$ e menor o valor de L. Isso decorre do fato de que a $\rho$ calculada com a equação (9) reflete, basicamente, o inverso do tamanho pretendido para a janela de tempo de níveis de $\mathrm{R}$ abaixo dos estabelecidos. Para que a janela se amplie, há necessidade de redução da $\rho$. A equação (9) pode ser usada então para calcular a diminuição de $\rho$ requerida num solo compactado, a fim de garantir a janela de tempo de níveis de $\mathrm{R}$ abaixo dos estabelecidos.

O cálculo da $\rho$ com a equação (9) leva em conta algumas das circunstâncias meteorológicas que determinam o regime hídrico e a capacidade de o fluxo de água no solo suprir a demanda da planta. A descrição matemática desses processos é complexa (JONG VAN LIER et al., 2008). Na equação (9), não há descrição do crescimento radicular nem da extração de água do solo no tempo. A representação desses processos foi feita por valores de $\mathrm{L}$ e $\mathrm{E}_{\mathrm{m}}$ sem 
dependência do tempo, o que está em desacordo com o curso natural desses processos. Contudo, o uso da equação (9) com valores de $\mathrm{L}$ e $\mathrm{E}_{\mathrm{m}}$ dentro de seus limites físicos é válido para generalizações acerca da circunstancialidade da densidade crítica.

Com a equação (8), pode-se analisar a mudança na resistência mecânica em função da evapotranspiração, profundidade do sistema radicular, tempo e densidade do solo. De maneira pontual, podese avaliar a dinâmica da $\mathrm{R}$ num solo com densidade igual à $\rho_{c}$. Para a $\rho_{c}$ do solo deste estudo $\left(\rho_{c}=1340 \mathrm{~kg}\right.$ $\mathrm{m}^{-3}$, correspondente ao teor de argila de $\left.0,640 \mathrm{~kg} \mathrm{~kg}^{-1}\right)$, calculada com a equação de REICHERT et al. (2009), a simulação indica que, após 6 dias de extração de água a taxas de $0,006 \mathrm{~m} \mathrm{~d}^{-1}$, a R pode aumentar de 3 para $4 \mathrm{e}$ chegar a $8 \mathrm{MP}$ se a profundidade do sistema radicular for 0,$6 ; 0,4$ ou 0,2m, respectivamente (Figura 1c). Porém, se a taxa de extração de água diminuir para $0,002 \mathrm{~m}$ $\mathrm{d}^{-1}$, após o sexto dia, a $\mathrm{R}$ aumenta de 2,7MP para apenas $3,4 \mathrm{MP}$ com o decréscimo de $0,6 \mathrm{~m}$ para $0,2 \mathrm{~m}$ da profundidade do sistema radicular.

As simulações deste estudo apontam situações em queplantas podem produzirnormalmente, mesmo que a densidade do solo seja igual ou maior que a $\rho_{c}$, o que tem sido verificado (COLLARES et al., 2006; KLEIN \& CAMARA, 2007; GUBIANI, 2012). Assim, as simulações deste estudo, associadas com evidências de pesquisas, indicam que é oportuno investigar se respostas de plantas em desacordo com o esperado para dado estado de compactação estão condicionadas por pequena evapotranspiração e sistema radicular profundo, fatores controladores do estresse hídrico (CARLESSO, 1995) e mecânico (WHITMORE \& WHALLEY, 2009). A confirmação dessa hipótese implicará a necessidade de descrever a dependência dos índices físicos do solo aos fatores meteorológicos, a fim de avaliar os riscos de perda na produção das culturas por compactação do solo.

\section{CONCLUSÃO}

As equações apresentadas neste trabalho possibilitaram calcular a densidade crítica do intervalo hídrico ótimo, a dinâmica da resistência mecânica e o decréscimo necessário de densidade para garantir que a resistência mecânica não ultrapasse níveis máximos estabelecidos. A diminuição da evapotranspiração e o aumento da espessura da camada de solo ocupada pelo sistema radicular retardam o aumento da resistência mecânica do solo e indicam circunstâncias para produção normal das plantas, mesmo que a densidade do solo seja igual à densidade crítica do intervalo hídrico ótimo.

\section{AGRADECIMENTOS}

Ao Conselho Nacional de Desenvolvimento Científico e Tecnológico (CNPq), pelo suporte financeiro (Edital $\mathrm{MCT} / \mathrm{CNPq} \mathrm{N}$ o 014/2010 - Universal).

\section{REFERÊNCIAS}

BUSSCHER, W.J. Adjustment of flat-tipped penetrometer resistance data to a common water content. Transactions of the ASAE, v.33, n.2, p.519-524, 1990.

CARLESSO, R. Absorção de água pelas plantas: água disponível versus extraível e a produtividade das culturas. Ciência Rural, v.25, n.1, p.183-188, 1995. Disponível em: <http://www.scielo. br/scielo.php?pid $=$ S0103-84781995000100035\& $\mathrm{script}=\mathrm{sci}$ abstract\&tlng=pt $>$. Acesso em: 04 jan. 2013. doi: 10.1590/S010384781995000100035 .

COLLARES, G.L. et al. Qualidade física do solo na produtividade da cultura do feijoeiro num argissolo. Pesquisa Agropecuária Brasileira, v.41, n.11, p.1663-1674, 2006. Disponível em: $<$ http://www.scielo.br/scielo.php?script=sci_arttext\&pid=S0100204X2006001100013 >. Acesso em: 04 jan. 2013. 10.1590/S0100$204 X 2006001100013$.

GUBIANI, P.I. Regularidade de resposta da cultura do milho à compactação do solo. 2012. 154f. Tese (Doutorado em Ciência do Solo) - Curso de Pós-graduação em Ciência do Solo, Universidade Federal de Santa Maria, RS.

JONG VAN LI ER et al. Macroscopic root water uptake distribution using a matric flux potential approach. Vadose Zone Journal, v.7 n.3, p.1065-1078, 2008. Disponível em: <https:/www.soils.org/ publications/vzj/abstracts/7/3/1065>. Acesso em: 04 jan. 2013. doi: $10.2136 /$ vzj2007.0083.

KAISER, D.R. et al. Intervalo hídrico ótimo no perfil explorado pelas raízes de feijoeiro em um latossolo sob diferentes níveis de compactação. Revista Brasileira de Ciência do Solo, v.33, n.4, p.845-855, 2009. Disponível em: <http://www.scielo.br/scielo. php?script $=$ sci_arttext\&pid $=$ S0100-06832009000400009\&lng =pt\&nrm=iso $>$. Acesso em: 04 jan. 2013. doi: 10.1590/S010006832009000400009 .

KLEIN, V.A.; CAMARA, R.K. Rendimento da soja e intervalo hídrico ótimo em Latossolo Vermelho sob plantio direto escarificado. Revista Brasileira de Ciência do Solo, v.31, n.2, p.221-227, 2007. Disponível em: <http://www.scielo.br/scielo.php?script=sci arttex t\&pid=S0100-06832008000100001>. Acesso em: 04 jan. 2013. doi: 10.1590/S0100-06832007000200004.

LETEY, J. Relationship between soil physical properties and crop productions. Advances in Soil Science, v.1, n., p.277-294, 1985.

REICHERT, J.M. et al. Reference bulk density and critical degreeof-compactness for no-till crop production in subtropical highly weathered soils. Soil \& Tillage Research, v.102, n.2, p.242-254, 2009. Disponível em: <http://www.sciencedirect.com/science/ article/pii/S0167198708001025>. Acesso em: 04 jan. 2013. doi: 10.1016/j.still.2008.07.002. 
REICHERT, J.M. et al. Qualidade dos solos e sustentabilidade de sistemas agrícolas. Revista Ciência \& Ambiente, v., n.27, p.2948,2003

SILVA, A.P. da et al. Characterization of the least limiting water range of soils. Soil Science Society of America Journal, v.58, n.6, p.1775-1781, 1994.

SILVA, A.P. da et al. Funções de pedotransferência para as curvas de retenção de água e de resistência do solo à penetração. Revista Brasileira de Ciência do Solo, v.32, n.1, p.1-10, 2008. Disponível em: <http://www.scielo.br/scielo.php?script=sci_arttext \&pid=S0100-06832008000100001>. Acesso em: 04 jan. 2013. doi: 10.1590/S0100-06832008000100001.

WHITMORE, A.P.; WHALLEY, W.R. Physical effects of soil drying on roots and crop growth. Journal of Experimental
Botany, v.60, n.10, p.2845-2857, 2009. Disponível em: <http:// jxb.oxfordjournals.org/content/60/10/2845.full>. Acesso em: 04 jan. 2013. doi: 10.1093/jxb/erp200.

TAVARES FILHO, J. et al. Modelling of soil penetration resistance for an oxisol under no-tillage. Revista Brasileira de Ciência do Solo, v.32, n.1, p. 86-95, 2012. Disponível em: $<$ http://www.scielo.br/scielo.php?script=sci_arttext\&pid=S010006832012000100010\&lng=pt\&nrm=iso $>$. Acesso em: 29 maio, 2013. doi: 10.1590/S0100-06832012000100010.

TWARAKAVI, N.K.C.; SAKAI, M. Jirka Šimůnek. An objective analysis of the dynamic nature of field capacity. Water Resources Research, v.45, n.10, p.1-9, 2009. Disponível em: <http:// onlinelibrary.wiley.com/doi/10.1029/2009WR007944/abstract>. Acesso em: 28 ago. 2013. doi: 10.1029/2009WR007944.

Ciência Rural, v.44, n.6, jun, 2014. 